\title{
PERFECT BLANKETS
}

\author{
BY \\ ANTHONY P. MORSE
}

1. Introduction. In a recent paper $\left(^{1}\right)$ A. S. Besicovitch made the interesting discovery that concentric circles about a point play an important role in the theory of covering and differentiation. On this account it is rather natural to hope that a similar role is played by moderately oblate convex sets which are associated with a point well inside them. The validity of some conjectures of this sort will be established. However, some other natural conjectures which at first glance seem, by continuity, to be almost immediate consequences of Besicovitch's paper are in reality false. Because of this, Theorem 5.13 is, in a more or less reasonable sense, the best of its kind.

In $\$ 2$ we discuss the value of certain blankets in connection with covering and differentiation. Perfect blankets are defined; their existence in general spaces and their importance are noted.

In $\$ 3$ we formulate and examine a fairly well known sufficient condition for the $\phi$ Vitali property.

In $\$ 4$ certain basic existence theorems are established.

We feel that the tools forged in $\$ 3$ and especially in $\$ 4$ have a wider use than the rather special one to which they are put in $\$ 5$.

By restricting ourselves in $\$ 5$ to Euclidean space of $n$ dimensions we see that a rather universal covering and differential theory results from associating certain star shaped sets with a point. In this way we extend the already mentioned concentric circle discovery of Besicovitch.

Readers primarily interested in this extension are advised to subject $\S \S 1$, 2,3 , and 4 to cursory examination and then begin reading $\$ 5$.

We assemble now a number of notations, conventions, definitions, and assumptions.

It is agreed that: $(x \in A)=(x$ is a member of $A)=(x$ is in $A) ;(x \notin A)=(x$ is not a member of $A)=(x$ is not in $A) ;(A \subset B)=(A$ is contained in $B)=(A$ is a subset of $B)=(B \supset A)=(B$ contains $A)$; the integer 0 and the vacuous set are equal; a family $B$ is disjointed if and only if $B_{1} B_{2}=0$ whenever $B_{1}$ and $B_{2}$ are different members of $\$ B$. A family is disjointed, in other words, if and only if no two different members of it intersect.

If $a_{x}$ is a set for each $x$ in a set $B$, then

$$
\sum_{x \in B} a_{x}=\underset{y}{E}\left[y \in a_{x} \text { for some } x \text { in } B\right] .
$$

Presented to the Society, November 2, 1946; received by the editors June 2, 1946.

(1) A. S. Besicovitch, A general form of the covering principle and relative differentiation of additive functions, Proc. Cambridge Philos. Soc. vol. 41 (1945) pp. 103-110. 
If $\mathfrak{F}$ is a family, then

$$
\sigma(\mathfrak{F})=\sum_{\beta \in \mathfrak{F}} \beta .
$$

Thus $x \in \sigma(\mathfrak{F})$ if and only if $x$ is a member of some member of $\mathfrak{F}$.

If $a_{x} \geqq 0$ for $x$ in a countable set $B$, then

$$
\sum_{x \in B} a_{x}
$$

is also the appropriate numerical sum. Whether numerical summation or set theoretic summation is intended in a given instance will be obvious from the context.

In addition we agree upon the following definition.

1.1. Definition.

$$
\operatorname{sng} x=\underset{y}{E}[y=x] \text {. }
$$

Thus sng $x$ is the set whose sole member is $x$.

We fix throughout the paper a space $\mathcal{S}$ metrized with $\rho$. More specifically we assume henceforth that $\rho$ is such a function on $E_{(x, y)}[(x \in \mathcal{S}),(y \in \mathcal{S})]$ that $\rho(x, x)=0 \leqq \rho(x, z) \leqq \rho(x, y)+\rho(z, y)<\infty$ whenever $x, y$, and $z$ are in $\delta$. It follows that $\rho(x, y)=\rho(y, x)$ for $x$ and $y$ in $S$, but it does not follow that $x=y$ whenever $\rho(x, y)=0$. Hereafter such words as "closed," "open," "Borel," "bounded," and so on refer to this metric $\rho$. In $\$ 5$ we shall be forced to assume much more about $\rho$.

1.2. Definition. For $A \subset S$ we define:

$$
\begin{array}{ll}
\operatorname{diam} A=\sup _{x \in A, \nu \in A} \rho(x, y) & \text { whenever } A \neq 0 ; \\
\operatorname{diam} A=0 & \text { whenever } A=0 .
\end{array}
$$

\subsection{Definition.}

$$
\mathfrak{S}(x, r)=\underset{y}{E}[\rho(x, y) \leqq r] .
$$

Thus $\mathfrak{}(x, r)$ is the closed sphere with $x$ as center and radius $r$.

1.4. Definition. The internal radius at $x$ of $\beta$ is the sup of those numbers $r$ for which $\mathfrak{E}(x, r) \subset \beta$. of $A$.

Consequently $\mathfrak{S}(x, s) \subset A$ whenever $s$ is less than the internal radius at $x$

Of interest to us are certain measures.

1.5. Definition. $\phi$ measures $S$ if and only if $\phi$ is such a function on $E_{\beta}[\beta \subset S]$ to $E_{t}[0 \leqq t \leqq \infty]$ that

$$
\phi(A) \leqq \sum_{\beta \in \mathfrak{F}} \phi(\beta)
$$

whenever $\mathfrak{F}$ is a countable family for which $A \subset \sigma(\mathfrak{F}) \subset \mathrm{S}$. 
Accordingly if $\phi$ measures $S$ then:

I. $\phi(0)=0$;

II. $\phi(B) \leqq \phi(A)$ whenever $B \subset A \subset S$;

III. $\phi\{\sigma(\mathfrak{F})\} \leqq \sum_{\beta \in \mathfrak{F}} \phi(\beta)$ whenever $\mathfrak{F}$ is a countable family for which $\sigma(\mathfrak{F}) \subset \mathrm{S}$.

1.6. Definition. The family $\mathfrak{U}$ is defined by: $\phi \in \mathfrak{U}$ if and only if $\phi$ so measures $S$ that

IV. $\phi(A+B)=\phi(A)+\phi(B)$ whenever

$$
\inf _{x \in A, y \in B} \rho(x, y)>0 .
$$

\subsection{Definition.}

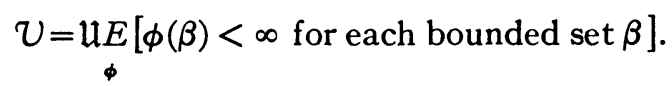

1.8. Definition. The family $\mathfrak{B}$ is defined by: $\phi \in \mathfrak{W}$ if and only if $\phi$ is such a member of $\mathfrak{U}$ that:

$\mathrm{V}$. Corresponding to each subset $\alpha$ of $\delta$ there is Borel set $\beta$ for which

$$
\alpha \subset \beta, \quad \phi(\alpha)=\phi(\beta) .
$$

1.9. Definition. $\mathscr{W}=\bigcup \mathfrak{B}$.

Due to Carathéodory $\left({ }^{2}\right)$ is the following definition.

1.10. Definition. $A$ is $\phi$ measurable if and only if $\phi$ so measures $\delta$ that

$$
\phi(T)=\phi(T A)+\phi(T-A)
$$

for each subset $T$ of $S$.

Clearly $\mathscr{W} \subset \mathcal{V} \subset \mathfrak{U}$. Information concerning measures in $\mathfrak{U}$ can be found in \$3. of a paper $\left.{ }^{3}\right)$ by Randolph and myself and also on pp. 424-444 of H. Hahn, Theorie der reellen Funktionen, vol. 1, Berlin, 1921.

1.11. Definition. $\mathfrak{F}$ covers $A$ if and only if $A \subset \sigma(\mathfrak{F})$.

1.12. Definition. $\mathfrak{F}$ covers $\phi$ almost all of $A$ if and only if

$$
\phi\{A-\sigma(\mathfrak{F})\}=0 .
$$

2. Perfect blankets. Of particular interest to us are certain blankets.

2.1. Definition. $F$ is a blanket if and only if $F$ is such a function that for each $x$ in its domain:

(i) $x \in \mathcal{S}$ and $F(x)$ is a family of bounded subsets of $S$;

(ii) $\inf _{\beta \in F(x)} \operatorname{diam}(\beta+\operatorname{sng} x)=0$.

Evidently the domain of a blanket is a subset of $S$.

2.2. Definition. If $F$ is a blanket with domain $A$ then the set

(2) C. Carathéodory, Über das lineare Mass von Punktmengen, Nachr. Ges. Wiss. Göttingen (1914) pp. 404-426.

(3) A. P. Morse and J. F. Randolph, The $\phi$ rectifiable subsets of the plane, Trans. Amer. Math. Soc. vol. 55 (1944) pp. 236-305. 


$$
\sum_{x \in A} F(x)
$$

is the spread of $F$.

2.3. Definition. $G$ is a subblanket of $F$ if and only if $G$ and $F$ are such blankets that: the domain of $G$ is contained in the domain of $F ; G(x) \subset F(x)$ for each $x$ in the domain of $G$.

Clearly the spread of $G$ is contained in the spread of $F$ whenever $G$ is a subblanket of $F$.

2.4. Definition. $F$ is a Borelian blanket if and only if $F$ is such a blanket that each member of its spread is a Borel set.

2.5. Definition. $F$ is a $\phi$ heavy blanket if and only if $F$ is a Borelian blanket, $\phi$ belongs 1.7 to $U$, and the spread of $F$ contains a countable disjointed family which covers $\phi$ almost all of the domain of $F$.

2.6. Definition. $F$ is a $\phi$ strong blanket if and only if $F$ is such a blanket that each of its subblankets is a $\phi$ heavy blanket.

2.7. Definition. $F$ is a perfect blanket if and only if $F$ is a $\phi$ strong blanket for each $\phi$ in $U$.

If $A$ is a separable set then in the sense of Definition 10.2 of my paper( $\left.{ }^{4}\right)$, $A$ theory of covering and differentiation, we can select a family $\mathfrak{F}$ which enmeshes $A$, and then by defining $F$ in accordance with $10.4 \mathrm{M}$ we see in the light of $10.5 \mathrm{M}$ and 2.7 that this $F$ is a perfect blanket with domain $A$. Thus if $\mathcal{S}$ itself is separable there is a perfect blanket with domain $S$.

2.8. Definition. The spherical blanket is the blanket $F$ such that, for each $x$ in $\mathcal{S}, F(x)$ is the family of spheres of the 1.3 form $\mathbb{S}(x, r)$ where $0<r<\infty$.

Several questions now arise. What conditions imposed on $\rho$ will insure the perfection of the spherical blanket? What conditions are imposed on $\rho$ by the assumed perfection of the spherical blanket? If the spherical blanket is perfect under some such assumption as compactness then of what sort of perfect blankets is the spherical blanket a special case? To none of these questions do I know the answer. In fact, at present, the only perfect blankets which I can associate with a general compact space are of the mesh type described above.

An understanding of the rest of this section is unessential to an understanding of the main results in the sections which follow.

The next four definitions will facilitate our discussion of derivatives.

2.9. Definition. $(F \boxminus \beta)$ is the family defined by: $G \in(F \boxminus \beta)$ if and only if $G$ is such a subblanket of $F$ that the domain of $G$ is the intersection of $\beta$ with the domain of $F$.

2.10. Definition. $f$ is $\phi$ addivelous with respect to $F$ if and only if $f$ is

(4) A. P. Morse, $A$ theory of covering and differentiation, Trans. Amer. Math. Soc. vol. 55 (1944) pp. 205-235. Hereinafter this reference is $M$. 
a function, $F$ is a blanket, and corresponding to each positive $\epsilon$, each $\beta$ in the spread of $F$, and each subblanket $F^{\prime}$ of $F$, there is a set $\alpha$ and an $F^{\prime \prime}$ in $\left(F^{\prime} \boxminus \alpha\right)$ for which:

$$
0 \leqq f(\beta)<\infty ; \quad \phi(\beta-\alpha)=0 ; \quad \sum_{B \in \Theta} f(B) \leqq f(\beta)+\epsilon
$$

whenever $B$ is a countable disjointed subfamily of the spread of $F^{\prime \prime}$.

2.11. Definition. $f$ is perfectly addivelous with respect to $F$ if and only if $f$ is a function, $F$ is a blanket, and corresponding to each positive $\epsilon$, each $\beta$ in the spread of $F$, and each subblanket $F^{\prime}$ of $F$, there is an $F^{\prime \prime}$ in $\left(F^{\prime} \varpi \beta\right)$ for which:

$$
0 \leqq f(\beta)<\infty ; \quad \sum_{B \in \mathcal{O}} f(B) \leqq f(\beta)+\epsilon
$$

whenever $(B)$ is a countable disjointed subfamily of the spread of $F^{\prime \prime}$.

In our next definition we define a derivative in terms of the limit notation set up in $2.9 \mathrm{M}$. To bring $2.9 \mathrm{M}$ into notational accord with the present paper replace in $2.9 \mathrm{M}$ the expression " $\{x\}$ " by the 1.1 expression "sng $x$ ".

2.12. Definition.

$$
\mathbf{D}(f, \phi, F, x)=\operatorname{limal}_{F(x) \ni \beta \rightarrow x} \frac{f(\beta)}{\phi(\beta)} .
$$

An evident consequence of 2.11 and 2.10 is the following theorem.

2.13. THEOREM. If $f$ is perfectly addivelous with respect to $F$ then, for each $\phi$ in $U, f$ is $\phi$ addivelous with respect to $F$.

It is possible, in a variety of ways, to come upon functions which are perfectly addivelous with respect to blankets. Perhaps one of the most important ways is given by the following theorem.

2.14. Theorem. If $F$ is a Borelian blanket and $f \in V$ then $f$ is perfectly addivelous with respect $F$.

Proof. Let $\epsilon$ be any positive number, let $\beta$ be any member of the spread of $F$, let $F^{\prime}$ be any subblanket of $F$. Since the bounded 2.1(i) Borel set $\beta$ is contained in a bounded open set we $\operatorname{can}\left({ }^{5}\right)$ and do choose an open set $\alpha$ for which

$$
\beta \subset \alpha, \quad f(\alpha) \leqq f(\beta)+\epsilon .
$$

Now let $A$ be the domain of $F^{\prime}$ and let $F^{\prime \prime}$ be such a blanket with domain $\beta A$ that

$$
F^{\prime \prime}(x)=F^{\prime}(x) \underset{\beta}{E}[\beta \subset \alpha]
$$

for each $x$ in $\beta A$. Clearly $F^{\prime \prime}$ is a blanket belonging to the 2.9 family $\left(F^{\prime} \varpi \beta\right)$.

(5) With the help of Theorem 4.10 in my paper, The role of internal families in measure theory, Bull. Amer. Math. Soc. vol. 50 (1944) p. 727. 
Moreover each member of the spread of $F^{\prime \prime}$ is contained in $\alpha$. Consequently

$$
\sum_{B \in \mathscr{S}} f(B) \leqq f(\alpha) \leqq f(\beta)+\epsilon
$$

whenever $\&$ is a countable disjointed subfamily of the spread of $F^{\prime \prime}$.

According to 2.11 the proof is complete.

By making those minor changes in the proof of $8.5 \mathrm{M}$ which are necessitated by our 2.10 revision of $8.3 \mathrm{M}$, we infer from $8.10 \mathrm{M}$ the following theorem.

2.15. Theorem. If $F$ is $a \phi$ strong blanket with domain $A$ and if $f$ is $\phi$ addivelous with respect to $F$, then

$$
0 \leqq \mathbf{D}(f, \phi, F, x)<\infty
$$

for $\phi$ almost all $x$ in $A$.

In consequence of 2.15 and 2.13 , and in contrast to 2.15 we obtain the following theorem.

2.16. TheOREM. If $F$ is a perfect blanket with domain $A$, and if $f$ is perfectly addivelous with respect to $F$, then for each $\phi$ in $U$,

$$
0 \leqq \mathrm{D}(f, \phi, F, x)<\infty
$$

for $\phi$ almost all $x$ in $A$.

The above theorem brings out an important attribute of perfect blankets. From 2.16 and 2.14 follows another theorem.

2.17. Theorem. If $F$ is a perfect blanket with domain $A$, then, for each $\phi$ in $U$, and each $f$ in $U$,

$$
0 \leqq \mathbf{D}(f, \phi, F, x)<\infty
$$

for $\phi$ almost all $x$ in $A$.

In Theorem 2.19 below we give another important property of perfect blankets.

2.18. Definition. $f$ is $\phi$ conservative if and only if $f(A)=0$ whenever $\phi(A)=0$.

Thus if $\phi \in \mathscr{W}, f \in \mathcal{W}, f(\mathcal{S})<\infty$, then a necessary and sufficient condition that $f$ be $\phi$ conservative is that $f$ be $\phi$ absolutely continuous.

2.19. ThEOREM. If $F$ is a perfect blanket with domain $\delta, f \in \mathcal{U}, \phi \in W$,

$$
B=\underset{x}{E}[\mathbf{D}(f, \phi, F, x)=\infty]
$$

then a necessary and sufficient condition that $f$ be $\phi$ conservative is that $f(B)=0$.

Because of our strong hypotheses the proof, which we omit, is not too difficult. It is interesting however that if we weaken our hypotheses either 
by 1.9 replacing " $\phi \in \mathscr{W}$ " by " $\phi \in U$ " or by replacing "perfect" by " $\phi$ strong," the resulting statement is false. The assumption that $\phi \in \mathscr{W}$ is used to show (cf. our proof of 2.14) that each set of $\phi$ measure 0 is contained in an open set whose $\phi$ measure is small. The assumption that $F$ is perfect is used to show that $F$ is $f$ strong.

The following definition and theorems are extracted from lectures given by me, in the Spring of 1943 , at the University of California.

2.20. Definition. $f$ is continuously $\phi$ addivelous with respect to $F$ if and only if $f$ is $\phi$ addivelous with respect to $F$ and

$$
f(\beta) \leqq \sum_{B \in \Theta} f(B)
$$

whenever $\beta$ is in the spread of $F$ and $B$ is such a countable disjointed subfamily of the spread of $F$ that $\phi\{\beta-\sigma(\mathbb{S})\}=0$.

2.21. THEOREM. If $F$ is a $\phi$ strong blanket with domain $\mathcal{S}, f$ is $\phi$ addivelous with respect to $F$, then

$$
\int_{\beta} \mathbf{D}(f, \phi, F, x) d \phi x \leqq f(\beta)
$$

or each $\beta$ in the spread of $F$.

2.22. Theorem. If $F$ is a $\phi$ strong blanket with domain $\mathcal{S}, f$ is $\phi$ addivelous with respect to $F$, then a necessary and sufficient condition that $f$ be continuously $\phi$ addivelous with respect to $F$ is that

$$
\int_{\beta} \mathbf{D}(f, \phi, F, x) d \phi x=f(\beta)
$$

or each $\beta$ in the spread of $F$.

In connection with 2.22 we point out that each $\phi$ conservative member of $U$ is clearly continuously $\phi$ addivelous with respect to each Borelian blanket.

2.23. Theorem. If $\phi \in W$, $F$ is a $\phi$ strong blanket with domain $\mathrm{S}$,

$$
-\infty<f(\beta)=\int_{\beta} u(x) d \phi x<\infty
$$

for each bounded $\phi$ measurable set $\beta$, then

$$
\mathrm{D}(f, \phi, F, x)=u(x)
$$

for $\phi$ almost all $x$.

If in 2.23 the assumption " $\phi \in \mathscr{W}$ " is struck out, then the resulting statement is false.

Theorem 2.17 suggests the following definition. 
2.24. Definition. $F$ is a universal blanket if and only if $F$ is such a blanket that whenever $f \in U$ and $\phi \in V$, then

$$
0 \leqq \mathbf{D}(f, \phi, F, x)<\infty,
$$

for $\phi$ almost all $x$ in the domain of $F$.

A perfect blanket is 2.17 clearly a universal blanket.

2.25. Remark. Although 2.17 is adequate for many differential purposes, situations nevertheless arise in which a direct appeal to 2.16 is advantageous. We give here an example of such a situation.

We suppose $S$ is the ordinary Euclidean plane and that $\rho$ is the customary metric. Let $F$ be any perfect blanket with domain $S$. Let $L$ be Carathéodory $\left({ }^{2}\right)$ linear measure. Let $C$ be any set for which $L(C)<\infty$. Let $b$ be any straight line and for $A \subset S$ let $A^{*}$ be the orthogonal projection of $A$ on $b$. Also let $\psi, p$, and $f$ be such functions on all subsets of $S$ that

$$
\begin{gathered}
\psi(A)=L(A C), \quad p(A)=L\left\{(A C)^{*}\right\}, \\
f(A)=\psi(A)-p(A),
\end{gathered}
$$

whenever $A \subset \mathrm{S}$.

Of course $\psi$ is a member of $V$, but in general $p$ and $f$ are not. However since the $L$ measure of a set is never increased by orthogonal projection on a line we are sure first of all that $0 \leqq f(A) \leqq \psi(A)<\infty$ whenever $A \subset \mathrm{S}$. Moreover by proceeding in much the same manner as we did in the proof of 2.14 we encounter little difficulty in verifying that $f$ is perfectly addivelous with respect to $F$.

Suppose now $\phi$ is any member of $U$. According to 2.17 and 2.16 we know :

$$
\begin{array}{ll}
0 \leqq \mathrm{D}(\psi, \phi, F, x)<\infty & \text { for } \phi \text { almost all } x ; \\
0 \leqq \mathrm{D}(f, \phi, F, x)<\infty & \text { for } \phi \text { almost all } x .
\end{array}
$$

By subtracting we learn

$$
0 \leqq \mathrm{D}(p, \phi, F, x)<\infty \quad \text { for } \phi \text { almost all } x .
$$

In particular we see:

$$
\begin{aligned}
& 0 \leqq \mathbf{D}(p, \psi, F, x)<\infty \quad \text { for } \psi \text { almost all } x ; \\
& 0 \leqq \mathbf{D}(p, \psi, F, x)<\infty \quad \text { for } L \text { almost all } x \text { in } C .
\end{aligned}
$$

\section{The $\phi$ Vitali property.}

3.1. Definition. $\mathfrak{F}$ has the $\phi$ Vitali property with respect to $A$ if and only if $\mathfrak{F}$ is such a family of $\phi$ measurable sets that corresponding to each finite disjointed subfamily $\mathfrak{S}$ of $\mathfrak{F}$ there is a countable disjointed family $\mathbb{B}$ for which

$$
\mathfrak{S} \subset \mathfrak{S} \subset \mathfrak{F}, \quad \phi\{A-\sigma(\mathfrak{S})\}=0 .
$$

Since every family of $\phi$ measurable sets has the $\phi$ Vitali property with re- 
spect to the vacuous set we infer from $5.2 \mathrm{M}$ the validity of the following theorem.

3.2. THEOREM. If $\Omega$ is a countable family of sets with respect to each of which $\mathfrak{F}$ has the $\phi$ Vitali property then $\mathfrak{F}$ has the $\phi$ Vitali property with respect to $\sigma(\Omega)$

3.3. TheOREM. If

$$
A=\sum_{\lambda=1}^{\infty} S_{\lambda}
$$

and if for each positive integer $\lambda$ the family $\mathfrak{F}$ has the $\phi$ Vitali property with respect to $S_{\lambda}$ then $\mathfrak{F}$ has the $\phi$ Vitali property with respect to $A$.

Proof. Recall 1.1, let

$$
\Re=\sum_{\lambda=1}^{\infty} \operatorname{sng} S_{\lambda}
$$

check that $A=\sigma(\Re)$, and apply 3.2 .

3.4. TheOREM. If $\phi \in \mathcal{U}, 0<\eta<\infty, B \subset \mathcal{S}, \mathfrak{F}$ is such a family of closed sets that corresponding to each bounded open set $\alpha$ there is a countable disjointed subfamily $\Re$ of $\mathfrak{F}$ for which

$$
\sigma(\Omega) \subset \alpha, \quad \phi(B \alpha) \leqq \eta \phi\{\sigma(\Omega)\},
$$

then $\mathfrak{F}$ has the $\phi$ Vitali property with respect to $B$.

Proof. Since $S$ is a countable sum of bounded sets there is, in view of 3.2, no loss in generality in assuming, as we now do, that $B$ is bounded.

Let $\mathfrak{W}$ be a finite disjointed subfamily of $\mathfrak{F}$. Let $S$ be a bounded open set containing $B$. By induction define a sequence $G$ for which: $G_{0}=\mathfrak{W}$; and, for each positive integer $k, G_{k}$ is such a finite disjointed subfamily of $\mathfrak{F}$ that $\sigma\left(G_{k}\right)$ is contained in the bounded open set

$$
S-\sum_{j=0}^{k-1} \sigma\left(G_{i}\right)
$$

and

$$
\phi\left\{B \cdot\left[S-\sum_{j=0}^{k-1} \sigma\left(G_{j}\right)\right]\right\} \leqq 2 \eta \phi\left\{\sigma\left(G_{k}\right)\right\} .
$$

That $G$ can be so defined is an almost immediate consequence of our hypotheses.

Noting that

$$
\sigma\left(G_{j}\right) \sigma\left(G_{k}\right)=0
$$

whenever $j$ and $k$ are integers for which $0 \leqq j<k<\infty$, we let 


$$
\text { (S) }=\sum_{j=0}^{\infty} G_{j}
$$

and observe not only that $\mathbb{B}$ is a countable disjointed family for which $\mathfrak{S \subset} \subset \subset \mathfrak{F}$, but also that

$$
\sum_{j=0}^{\infty} \phi\left\{\sigma\left(G_{j}\right)\right\}=\phi\left\{\sum_{j=0}^{\infty} \sigma\left(G_{j}\right)\right\} \leqq \phi(S)<\infty .
$$

Consequently

$$
\lim _{k} \phi\left\{\sigma\left(G_{k}\right)\right\}=0
$$

For each positive integer $k$ :

$$
\begin{aligned}
B-\sigma(\mathbb{S}) & =B S-\sigma(\mathbb{S})=B \cdot[S-\sigma(\mathbb{S})] \\
& =B \cdot\left[S-\sum_{j=0}^{\infty} \sigma\left(G_{j}\right)\right] \subset B \cdot\left[S-\sum_{j=0}^{k-1} \sigma\left(G_{j}\right)\right] ;
\end{aligned}
$$

hence because of (1)

$$
\phi\{B-\sigma(())\} \leqq \phi\left\{B \cdot\left[S-\sum_{j=0}^{k-1} \sigma\left(G_{j}\right)\right]\right\} \leqq 2 \eta \phi\left\{\sigma\left(G_{k}\right)\right\} .
$$

This and (2) tell us

$$
\phi\{B-\sigma(\Im)\}=0 .
$$

Accordingly $\mathfrak{F}$ has the $\phi$ Vitali property with respect to $B$.

\section{Some existence theorems.}

4.1. Definition. We say $N$ is a nest whenever $N$ is such a family that if $H$ and $K$ are any member of $N$ then either $H \subset K$ or $K \subset H$.

Of use to us is the following lemma.

4.2. Lemma. If $\Omega$ is a family of sets then $\Omega$ contains such a nest $N$ that no member of $\Omega-N$ contains every member of $N$.

For references to the literature in connection with 4.2 see footnote 8 on page 208 of M. Some mathematicians regard 4.2 as self-evident. In case $\Omega$ is countable a proof might run something like this.

Arrange the members of $\Omega$ in a sequence. Let $S_{0}$ be the first member, $S_{1}$ be the first member which contains $S_{0}$ as a proper subset, and so on. The family whose members are $S_{0}, S_{1}, S_{2}, \ldots$ is evidently a nest of the desired sort.

Even though $\Omega$ is non-countable the above considerations give us some assurance of the existence of the required nest.

I prefer, with the help of 4.2 , to forge here certain general tools rather than, ignoring their existence, to attack directly, with the assistance of defini- 
tion by countable induction, the problems of $\S 5$. An examination of $\S 5$ and the rest of this section will reveal that a successful direct attack of this sort is indeed possible.

4.3. TeRminology. We agree that $R$ is a relation if and only if $R$ is a set of ordered pairs. As with functions it is understood that the domain of a relation $R$ consists of all those points $x$ such that there is a $y$ for which $(x, y) \in R$. Similarly the range of a relation $R$ consists of all those points $y$ such that there is an $x$ for which $(x, y) \in R$.

The fundamental property of ordered pairs is, of course, that $x=s$ and $y=t$ when, and only when, $(x, y)=(s, t)$.

4.4. Definition. $R$ is a covering relation if and only if $R$ is a relation and $x \in \alpha$ whenever $(x, \alpha) \in R$.

An example of this sort of relation is the set of ordered pairs of the form $(x, \mathfrak{S}(x, r))$ where $x \in \mathcal{S}$ and $0<r<\infty$.

4.5. Definition. $R$ is $\Delta$ restrained if and only if $\Delta$ is such a function and $R$ is such a covering relation that

$$
y \notin \alpha \text { and } \Delta(y, \beta) \leqq 2 \Delta(x, \alpha)
$$

whenever $(x, \alpha)$ and $(y, \beta)$ are two different members of $R$ for which $x \in \beta$.

4.6. THEOREM. If $Q$ is a covering relation with domain $A$ and if $\Delta$ is a nonnegative function which is bounded on $Q$, then $Q$ contains a $\Delta$ restrained relation whose range covers $A$.

Proof. Let

$$
H^{+}=Q \underset{(y, \beta)}{E}[y \notin \gamma \text { whenever }(z, \gamma) \text { is in } H]
$$

and observe

$$
H H^{+}=0 \quad \text { whenever } H \subset Q .
$$

Let $\Omega^{\prime}$ be the family of all $\Delta$ restrained subrelations of $Q$ and let $\Omega^{\prime \prime}$ be defined by: $H \in \Omega^{\prime \prime}$ if and only if $H$ is such a subset of $Q$ that

$$
\Delta(y, \beta) \leqq 2 \Delta(x, \alpha)
$$

whenever $(x, \alpha)$ is any member of $H$ and $(y, \beta)$ is any member of $H^{+}$. Let $\Omega=\Omega^{\prime} \Omega^{\prime \prime}$ and in accord with 4.2 select such a nest $N$ that $\Omega \supset N$ while no member of $\Omega-N$ contains every member of $N$. Let $R=\sigma(N)$.

It is easily checked that $R \in \Omega^{\prime}$, and, since $H^{+} \supset R^{+}$whenever $H \subset R$, it is also easily checked that $R \in \Omega^{\prime \prime}$. Thus $R \in \Omega$, and we shall know that $R$ is a subrelation of $Q$ of the required sort as soon as we have verified the following statement.

Statement. The range of $R$ covers $A$.

Proof. (By contradiction.) Suppose the range of $R$ does not cover $A$; note 
then that $R^{+} \neq 0$; invoke the boundedness of $\Delta$ to ascertain an $(X, \Gamma)$ in $R^{+}$for which

$$
\Delta(y, \beta) \leqq 2 \Delta(X, \Gamma) \quad \text { whenever }(y, \beta) \in R^{+} ;
$$

and 1.1 set $P=R+$ sng $(X, \Gamma)$.

Now if $(x, \alpha)$ and $(y, \beta)$ are different members of $P$ for which $x \in \beta$ then: necessarily $(x, \alpha) \in R$ and either $(y, \beta) \in R$ or $(y, \beta)=(X, \Gamma)$; in case $(y, \beta) \in R$ then the conclusion,

$$
y \notin \alpha \text { and } \Delta(y, \beta) \leqq 2 \Delta(x, \alpha),
$$

is a consequence of the fact that $R \in \Omega \subset \Omega^{\prime}$; whereas in case $(y, \beta)=(X, \Gamma)$ then it is a consequence of the fact that $(X, \Gamma) \in R^{+}$and $R \in \Omega \subset \Omega^{\prime \prime}$.

Accordingly $P \in \Omega^{\prime}$. Moreover, because $R^{+} \supset P^{+}$, it is easily seen in the light of (2) that $P \in \Omega^{\prime \prime}$. Thus $P$ is a member of $\Omega$ which contains every member of $N$. We recall our selection of $N$ and infer

$$
\begin{gathered}
P \notin \Omega-N, \quad P \in N, \quad P \subset \sigma(N)=R, \quad R+\operatorname{sng}(X, \Gamma) \subset R \\
\operatorname{sng}(X, \Gamma) \subset R, \quad(X, \Gamma) \in R .
\end{gathered}
$$

But, since $(X, \Gamma)$ is also in $R^{+}$we conclude $0 \neq R R^{+}$in contradiction to (1).

4.7. Definition. $R$ is diametrically restrained if and only if $R$ is a covering relation, each member of the range of $R$ is contained in $S$, and

$$
y \notin \alpha \text { and } \operatorname{diam} \beta \leqq 2 \operatorname{diam} \alpha
$$

whenever $(x, \alpha)$ and $(y, \beta)$ are two different members of $R$ for which $x \in \beta$.

4.8. THEOREM. If $Q$ is a covering relation with domain $A$ and range $\mathfrak{Q}$, and if $\sigma(\mathfrak{Q}) \subset \mathrm{S}$ with

$$
\sup _{\beta \in \mathbb{Q}} \operatorname{diam} \beta<\infty,
$$

then $Q$ contains a diametrically restrained relation whose range covers $A$.

Proof. Let $\Delta$ be the function on $Q$ such that

$$
\Delta(y, \beta)=\operatorname{diam} \beta \quad \text { for }(y, \beta) \in Q .
$$

Note that every $\Delta$ restrained subrelation of $Q$ is also diametrically restrained. Apply 4.6.

4.9. THEOREM. If $\mathfrak{F}$ is a family of non-vacuous subsets of $\mathcal{S}$ and $\Delta$ is a nonnegative function which is bounded on. F then $\mathfrak{F}$ contains such a disjointed family (S) that each $\beta$ in $\mathfrak{F}$ intersects some $B$ in $\mathbb{B}$ in such a way that $\Delta(\beta) \leqq 2 \Delta(B)$.

Proof. For $\mathfrak{S} \subset \mathfrak{F}$ let

$$
\mathfrak{S}^{+}=\mathfrak{F} \underset{\beta}{E}[\beta \sigma(H) \neq 0] .
$$


Let $\Omega$ be the family consisting of such disjointed subfamilies $\mathfrak{W}$ of $\mathfrak{F}$ that each $\beta$ in $\mathfrak{S}^{+}$intersects some $B$ in $\mathfrak{E}$ in such a way that $\Delta(\beta) \leqq 2 \Delta(B)$. Using 4.2 select such a nest $N$ that $\Omega \supset N$ while no member of $\Omega-N$ contains every member of $N$. Let $\$=\sigma(N)$.

Since $N$ is a nest and since

$$
\mathfrak{S}=\sum_{\mathfrak{S} \in N} \mathfrak{W} \subset \mathfrak{F}, \quad \mathfrak{S}^{+}=\sum_{\mathfrak{S} \in N} \mathfrak{W}^{+}
$$

it is clear that $\mathbb{B}$ is such a disjointed subfamily of $\mathfrak{F}$ that each $\beta$ in $\mathfrak{S}^{+}$intersects some $B$ in $B$ in such a way that $\Delta(\beta) \leqq 2 \Delta(B)$.

Thus completion of the proof hinges on the following statement.

STATEMENT. $\mathbb{B}+=\mathfrak{F}$.

Proof. (By contradiction.) Suppose $\mathfrak{S}^{+} \neq \mathfrak{F}$, let $\Re=\mathfrak{F}-\mathfrak{S}^{+}$, note that $\Re \neq 0$, and invoke the boundedness of $\Delta$ to so ascertain $b$ in $\Re$ that

$$
\Delta(\beta) \leqq 2 \Delta(b) \quad \text { whenever } \beta \in \Re .
$$

Inasmuch as $\$+$ sng $b$ is evidently a member of $\Omega$ which contains every member of $N$ we infer, because of our selection of $N$, that

$$
\text { B + sng } b \in N, \quad B+\operatorname{sng} b \subset \sigma(N)=\mathbb{B}, \quad b \in \mathbb{B} \text {. }
$$

Thus, since $B \subset B^{+}$, we conclude that $b \in B^{+}$in contradiction to the fact that $b \in \Re=\mathfrak{F}-\mathbb{S}^{+}$.

An immediate consequence of 4.9 is the following theorem.

4.10. THEOREM. If $\mathfrak{F}$ is a family of non-vacuous subsets of $\mathcal{S}$ and

$$
\sup _{\beta \in \mathfrak{F}} \operatorname{diam} \beta<\infty
$$

then $\mathfrak{F}$ contains such a disjointed family \& that each $\beta$ in $\mathfrak{F}$ intersects some $B$ in (B) in such a way that $\operatorname{diam} \beta \leqq 2 \operatorname{diam} B$.

5. Star blankets are perfect blankets. Throughout this section it is agreed that: $n$ is a positive integer; $S$ is ordinary Cartesian $n$ space with addition, subtraction, and multiplication conventionally defined and designated; $\theta$ is the origin of zero element of $S$; for $x$ in $S$

$$
\|x\|=\left\{\sum_{i=1}^{n} x_{i}^{2}\right\}^{1 / 2}
$$

where $x_{i}$ is the $i$ th coordinate of $x$;

$$
\rho(x, y)=\|x-y\|
$$

whenever $x \in \mathcal{S}$ and $y \in S$. Thus $\|x\|$ is the usual norm of $x$ and $\rho$ is the usual Euclidean metric for $S$. The special algebraic form of our norm is unimpor- 
tant; in the sequel we merely assume:

$$
\begin{aligned}
0 & =\|\theta\| ; \\
0 & <\|x\| \\
\|t x\| & =|t|\|x\| \\
\|x+y\| & \leqq\|x\|+\|y\|
\end{aligned}
$$

whenever $x \in S$ and $x \neq \theta$; whenever $x \in S$ and $-\infty<t<\infty$; whenever $x \in S$ and $y \in S$.

If $F$ is such a blanket that, for each $x \in \mathcal{S}, F(x)$ is the family of all those closed spheres of positive finite radius to which $x$ belongs then: if $n=1$ then, as is known, $F$ is a 2.7 perfect blanket and hence a 2.24 universal blanket; but, if $n=2$, then $F$ is not even universal. However, due to Besicovitch( $\left.{ }^{1}\right)$ is the interesting discovery, easily extended to higher dimensions, that the 2-dimensional spherical 2.8 blanket is perfect. One of the consequences $\left({ }^{6}\right)$ of this perfection is discussed in the following remark.

5.1. REMARK. Let $\phi$ be a member of $\mathcal{U}$, and let $1<\lambda<\infty$. Suppose $F$ is such a blanket with domain $S$ that, for $x \in \mathcal{S}, \beta \in F(x)$ when and only when $\beta$ is a closed set and there is a positive finite number $r$ for 1.3 which

$$
\beta \subset \mathfrak{S}(x, r) \text { and } \phi\{\mathfrak{S}(x, r)\} \leqq \lambda \phi(\beta) \text {. }
$$

Under these circumstances, according to $12.4 \mathrm{M}$, it is clear that $F$ is a $\phi$ strong blanket. From 2.15 differential properties of $F$ follow.

More specifically let us now assume $\phi$ is Lebesgue measure. Clearly the spherical blanket is a subblanket of $F$ and $F$ is of interest in connection with Lebesgue measure. In fact the classical results about differentiation with respect to regular families are at hand. Nevertheless a differential theory employing $F$ instead of the spherical blanket is greatly impoverished by the fact that $F$ is neither perfect nor universal. Of course these defects come as no great surprise inasmuch as our assumptions about $F$ involve $\phi$.

5.2. REMARK. Of what sort of perfect blankets, then, is the spherical blanket an example? One would guess that a close approximation to the spherical blanket would also be perfect. After recalling 1.4 let us so construct a blanket $F$ with domain $S$ that for each $x \in S$ :

$$
\underset{F(x) \exists \beta \rightarrow x}{\operatorname{limal}}(\operatorname{diam} \beta)(\text { internal radius at } x \text { of } \beta)^{-1}=2 \text {; }
$$

each member of $F(x)$ is not only closed but star shaped about $x$ as well. Thus for each $x \in \mathcal{S}$, as $F(x) \ni \beta \rightarrow x$, we see that $\beta$ contains one sphere about $x$ and is contained in a second sphere about $x$ with the ratio of their radii tending to 1. Accordingly $F$ is very much like the spherical blanket. Moreover it would seem that the perfection of the spherical blanket could be used in an argument involving continuity to establish the perfection of $F$. If $n=1$ then be-

(8) Cf. A. S. Besicovitch, $A$ general form of the covering principle ond relative differentiation of additive functions. II, Proc. Cambridge Philos. Soc. vol. 42 (1946) pp. 1-10. 
cause $F(x)$ consists of closed intervals to which $x$ belongs it follows that $F$ is a perfect blanket. However, if $n=2$ then it can be shown by an example, which is not given here, that our $F$ need not even be 2.24 universal. Nevertheless if we demand somewhat more of $F$ in the way of star shape and somewhat less of it in the way of sphere shape then $F$ will necessarily become perfect and hence universal.

To this modification we now attend.

5.3. Definition. For $\beta \subset S$ we agree that the $h u b$ of $\beta$ consists of those points $y$ in $\beta$ such that

$$
\{(1-t) x+t y\} \in \beta
$$

whenever $x \in \beta$ and $0 \leqq t \leqq 1$.

It is evident that the hub of a convex subset of $S$ is the set itself. Thus since the hub of any set is convex we see that a necessary and sufficient condition that a subset $\beta$ of $S$ be convex is that $\beta$ equal its hub. Also of some interest is the fact that the hub of any subset $\beta$ of $\delta$ is the intersection of all maximal convex subsets of $\beta$.

5.4. Definition. The hub radius at $x$ of $\beta=$ the 1.4 internal radius at $x$ of the hub of $\beta$.

5.5. AbBreviation. hbrd $(x, \beta)=$ the hub radius at $x$ of $\beta$.

In $2.9 \mathrm{M}$ is explained a typographical variant of the limit notation appearing in the following definition.

5.6. Definition. $F$ is a star blanket if and only if $F$ is a blanket and for each $x$ in its domain $F(x)$ is such a family of closed sets that

$$
\operatorname{limalsup}_{F(x) \ni \beta \rightarrow x} \frac{\operatorname{diam} \beta}{\operatorname{hbrd}(x, \beta)}<\infty .
$$

It is our aim to show that each star blanket is a perfect blanket. Such a demonstration would become impossible as was indicated in 5.2, if in 5.6 the expression "hbrd $(x, \beta)$ " were to be replaced by "the internal radius at $x$ of $\beta^{n}$. Should such a change be made an insurmountable obstacle would be met in any attempt to verify Part IX of Theorem 5.9. Of course in dealing with convexical blankets it should be borne in mind that the hub radius at $x$ of a convex set, $\beta$, is equal to the inner radius at $x$ of $\beta$.

As far as I can make out there is no way of deducing the perfection of star blankets from the perfection of the spherical blanket. However the spherical blanket is a star blanket. Aside from generality star blankets have a number of advantages not shared by the spherical blanket.

Firstly star blankets afford a great variety of difference quotients some of which may form a far better basis for computation than the corresponding spherical difference quotients.

Secondly if $T$ is a continuously differentiable one-to-one mapping, with nonvanishing Jacobian, of $S$ into itself then $T$ transforms, in the obvious 
sense, any star blanket into a star blanket. Such invariance is enjoyed, in general, by neither convexical star blankets nor the spherical blanket. In fact for the spherical blanket such invariance is, in general, clearly out of the question even when $T$ is restricted to be linear and nonsingular.

Thirdly star blankets are of use in establishing the almost everywhere validity of certain chain rules $\left({ }^{7}\right)$ of differentiation even though the rules themselves involve no blanket other than the spherical blanket.

5.7. Definition. For each set $A, \nu(A)$ is the number of points in $A$.

5.8. Definition. $\operatorname{circ} A$ is the family of all those sets $B$ which intersect $A$ in such a way that

$$
\operatorname{diam} A \leqq 2 \operatorname{diam} B \text {. }
$$

We shall use $4.3,4.7 .5 .5,5.7$ and 5.8 in the statement of the following theorem.

5.9. THEOREM. If $1 \leqq \lambda<\infty, a \in \Re, R$ is a diametrically restrained covering relation with range $\Re$, and

$$
\operatorname{diam} \beta<\lambda \operatorname{hbrd}(z, \beta)
$$$$
\text { whenever }(z, \beta) \in R \text {. }
$$

then $(8)$

$$
\nu(\Re \operatorname{circ} a)<(9 \lambda)^{3 n} .
$$

Proof. If $(x, \beta)$ and $(y, \beta)$ were different members of the diametrically restrained relation $R$ then, since $x \in \beta$ in accordance with 4.4, we 4.7 know that $y \notin \beta$ in contradiction to our 4.4 knowledge that $y \in \beta$. Therefore if $(x, \beta) \in R$ and $(y, \beta) \in R$ then we are compelled to grant that $(x, \beta)=(y, \beta)$ and hence that $x=y$. In other words, corresponding to each $\beta$ in $\Re$ there is precisely one $x$ for which $(x, \beta) \in R$; this $x$ it is herein agreed is $\beta^{\prime}$.

It is also agreed that:

$$
\begin{aligned}
\beta^{*} & =\beta^{\prime}-a^{\prime} ; \\
K_{\beta} & =\mathfrak{C}\left(\beta^{\prime},(4 \lambda)^{-1} \operatorname{diam} \beta\right) ; \\
\mathfrak{B} & =\Re \underset{\beta}{E}\left[\left\|\beta^{*}\right\| \leqq 8 \lambda \operatorname{diam} a\right] \operatorname{circ} a ; \\
\mathfrak{Q} & =\Re \underset{\beta}{E}\left[\left\|\beta^{*}\right\|>8 \lambda \operatorname{diam} a\right] \operatorname{circ} a ; \\
\mu(A) & =\text { the ordinary } n \operatorname{dimensional~Lebesgue~(outer)~measure~of~} A ; \\
v & =\mu\{\mathfrak{\complement}(\theta, 1)\} .
\end{aligned}
$$

With these conventions in mind we divide the remainder of the proof into 13 parts the first of which is well known and the second of which is evidently a consequence of (1).

${ }^{(7)}$ Cf. A. P. Morse, Convergence in variation and related topics, Trans. Amer. Math. Soc. vol. 41 (1937) p. 53, Theorem 3.1.

(8) No attempt is made to secure a precise estimate. 
PART I. If $0 \leqq r<\infty, z \in S$, then

$$
\mu\{\mathfrak{C}(z, r)\}=r^{n} v .
$$

PART II. If $\beta \in \Re$ then $\operatorname{diam} \beta<\lambda$ hbrd $\left(\beta^{\prime}, \beta\right)$.

PART III. If $\beta \in \Re$ then

$$
K_{\beta} \subset \mathbb{E}\left(\beta^{\prime}, \lambda^{-1} \operatorname{diam} \beta\right) \subset \beta .
$$

Proof. Use the definition of $K_{\beta}, 5.5,5.3$, and Part II.

From 4.7 and our hypotheses follows:

PART IV. If $\alpha$ and $\beta$ are such different members of $\Re$ that $\alpha^{\prime} \in \beta$ then

$$
\beta^{\prime} \notin \alpha \text { and } \operatorname{diam} \beta \leqq 2 \operatorname{diam} \alpha .
$$

PART V. If $\alpha$ and $\beta$ are such different members of $\Re$ that $\alpha^{\prime} \in \beta$ then

$$
\lambda^{-1} \operatorname{diam} \alpha<\left\|\beta^{\prime}-\alpha^{\prime}\right\| .
$$

Proof. Parts III and IV assure us

$$
\begin{gathered}
\mathfrak{C}\left(\alpha^{\prime}, \lambda^{-1} \operatorname{diam} \alpha\right) \subset \alpha, \quad \beta^{\prime} \notin \alpha, \\
\beta^{\prime} \notin \mathbb{C}\left(\alpha^{\prime}, \lambda^{-1} \operatorname{diam} \alpha\right), \quad\left\|\beta^{\prime}-\alpha^{\prime}\right\|>\lambda^{-1} \operatorname{diam} \alpha .
\end{gathered}
$$

PART VI. If $b$ and $\beta$ are such members of $\Re$ that $b^{\prime} \in \beta$ then

$$
\lambda^{-1} \operatorname{diam} b \leqq \operatorname{diam} \beta \leqq 2 \operatorname{diam} \cdot b .
$$

Proof. If $\beta \neq b$ then Part V implies that

$$
\lambda^{-1} \operatorname{diam} b<\left\|\beta^{\prime}-b^{\prime}\right\| \leqq \operatorname{diam} \beta,
$$

whereas Part IV implies that $\operatorname{diam} \beta \leqq 2 \operatorname{diam} b$. On the other hand if $\beta=b$ the conclusion is evident.

PART VII. If $\alpha$ and $\beta$ are different members of $\Re$ then

$$
(2 \lambda)^{-1} \operatorname{diam} \beta<\left\|\beta^{\prime}-\alpha^{\prime}\right\| \text {. }
$$

Proof. (By contradiction.) Suppose

$$
\left\|\beta^{\prime}-\alpha^{\prime}\right\| \leqq(2 \lambda)^{-1} \operatorname{diam} \beta .
$$

Reference to (2) and Part III informs us

$$
\alpha^{\prime} \in \mathbb{S}\left(\beta^{\prime},(2 \lambda)^{-1} \operatorname{diam} \beta\right) \subset \mathbb{E}\left(\beta^{\prime}, \lambda^{-1} \operatorname{diam} \beta\right) \subset \beta
$$

which in turn informs us $\alpha^{\prime} \in \beta$. In contradiction to (2) it may now be inferred from Part V and the replacement of " $b$ " by " $\alpha$ " in Part VI that

$$
\left\|\beta^{\prime}-\alpha^{\prime}\right\|>\lambda^{-1} \operatorname{diam} \alpha=(2 \lambda)^{-1}(2 \operatorname{diam} \alpha) \geqq(2 \lambda)^{-1} \operatorname{diam} \beta .
$$

PART VIII. If $\alpha$ and $\beta$ are different members of $\Re$ then

$$
K_{\alpha} K_{\beta}=0 \text {. }
$$


Proof. The supposition that $z \in K_{\alpha} K_{\beta}$ leads via Part VII to the two relations

$$
\begin{gathered}
\left\|\beta^{\prime}-z\right\| \leqq(4 \lambda)^{-1} \operatorname{diam} \beta<2^{-1}\left\|\beta^{\prime}-\alpha^{\prime}\right\|, \\
\left\|z-\alpha^{\prime}\right\| \leqq(4 \lambda)^{-1} \operatorname{diam} \alpha<2^{-1}\left\|\beta^{\prime}-\alpha^{\prime}\right\| .
\end{gathered}
$$

These combine to yield the contradiction $\left\|\beta^{\prime}-\alpha^{\prime}\right\|<\left\|\beta^{\prime}-\alpha^{\prime}\right\|$.

PART IX. If $b \in \mathfrak{Q} \operatorname{circ} a, \beta \in \mathfrak{Q} \operatorname{circ} a$

$$
\begin{aligned}
8 \lambda \text { diam } a<\left\|b^{*}\right\| & \leqq\left\|\beta^{*}\right\|, \\
\|\| \beta^{*}\left\|^{-1} \beta^{*}-\right\| b^{*}\left\|^{-1} b^{*}\right\| & \leqq(8 \lambda)^{-1},
\end{aligned}
$$

then $b^{\prime} \in \beta$.

Proof. Let

$$
s=\left.\left\|\beta^{*}\right\|\left\|b^{*}\right\|\right|^{-1}, \quad t=s^{-1}
$$

note that

$$
1 \leqq s<\infty ;
$$

observe 5.8 that $\beta a$ is nonvacuous and so choose $x$ that

$$
x \in \beta a ;
$$

agree

$$
y=(1-s) x+s b^{\prime}
$$

and check

$$
\begin{gathered}
0 \leqq t \leqq 1, \\
b^{\prime}=(1-t) x+t y .
\end{gathered}
$$

If $\left\|y-\beta^{\prime}\right\|<$ hbrd $\left(\beta^{\prime}, \beta\right)$ then, by 5.5 , the point $y$ belongs to the hub of $\beta$, and from (9), (7) and 5.3 it follows that $b^{\prime} \in \beta$. Thus the validity of Part IX is a consequence of the following statement.

Statement. $\left\|y-\beta^{\prime}\right\|<\operatorname{hbrd}\left(\beta^{\prime}, \beta\right)$.

Proof. In view of (8) and (5) we see

$$
\begin{aligned}
y-\beta^{\prime} & =(1-s) x+s b^{\prime}-\beta^{\prime} \\
& =(1-s)\left(x-a^{\prime}\right)+s\left(b^{\prime}-a^{\prime}\right)-\left(\beta^{\prime}-a^{\prime}\right) \\
& =(1-s)\left(x-a^{\prime}\right)+s b^{*}-\beta^{*} \\
& =(1-s)\left(x-a^{\prime}\right)+\left\|\beta^{*}\right\|\left\|b^{*}\right\|^{-1} b^{*}-\beta \\
& =(1-s)\left(x-a^{\prime}\right)+\left\|\beta^{*}\right\|\left\{\left.\left\|b^{*}\right\|\right|^{-1} b^{*}-\left\|\beta^{*}\right\|-1 \beta^{*}\right\} .
\end{aligned}
$$


From this and from (4), (6), (7), (3) and (5) we infer

$$
\begin{aligned}
\left\|y-\beta^{\prime}\right\| & \leqq|1-s|\left\|x-a^{\prime}\right\|+\left\|\beta^{*}\right\|(8 \lambda)^{-1} \\
& =(s-1)\left\|x-a^{\prime}\right\|+\left\|\beta^{*}\right\|(8 \lambda)^{-1} \\
& \leqq s \operatorname{diam} a+\left\|\beta^{*}\right\|(8 \lambda)^{-1} \\
& =(8 \lambda)^{-1}(s)(8 \lambda) \operatorname{diam} a+\left\|\beta^{*}\right\|(8 \lambda)^{-1} \\
& \leqq(8 \lambda)^{-1} s\left\|b^{*}\right\|+\left\|\beta^{*}\right\|(8 \lambda)^{-1} \\
& =(8 \lambda)^{-1}\left\|\beta^{*}\right\|+\left\|\beta^{*}\right\|(8 \lambda)^{-1} \\
& =(4 \lambda)^{-1}\left\|\beta^{*}\right\| .
\end{aligned}
$$

Having just shown

$$
\left\|y-\beta^{\prime}\right\| \leqq(4 \lambda)^{-1}\left\|\beta^{*}\right\|
$$

we next estimate $\left\|\beta^{*}\right\|$.

Because $\beta \in \operatorname{circ} a$ it is clear from 5.8 that $\operatorname{diam} a \leqq 2 \operatorname{diam} \beta$. Consequently [see (7)]

$$
\begin{aligned}
\left\|\beta^{*}\right\|=\left\|\beta^{\prime}-a^{\prime}\right\| \leqq\left\|\beta^{\prime}-x\right\|+\left\|x-a^{\prime}\right\| & \leqq \operatorname{diam} \beta+\operatorname{diam} a \\
& \leqq 3 \operatorname{diam} \beta \leqq 4 \operatorname{diam} \beta
\end{aligned}
$$

But since, by Part II,

$$
\operatorname{diam} \beta<\lambda \operatorname{hbrd}\left(\beta^{\prime}, \beta\right),
$$

we are now in a position to conclude from (10), (11) and (12) that

$$
\begin{aligned}
\left\|y-\beta^{\prime}\right\| & \leqq(4 \lambda)^{-1}\left\|\beta^{*}\right\| \leqq(4 \lambda)^{-1}(4) \operatorname{diam} \beta \\
& =\lambda^{-1} \operatorname{diam} \beta<\lambda^{-1} \lambda \operatorname{hbrd}\left(\beta^{\prime}, \beta\right)=\operatorname{hbrd}\left(\beta^{\prime}, \beta\right) .
\end{aligned}
$$

PART X. If diam $\alpha<(8 \lambda)^{-1}$ then

$$
\nu\left(\mathscr{\beta} \underset{\beta}{E}\left[\left\|\beta^{*}\right\|-1 \beta^{*} \in \alpha\right]\right) \leqq 8^{n} \lambda^{2 n} .
$$

The desired result is clearly a consequence of the following statement.

Statement. If $\mathfrak{W}$ is any finite subfamily of

$$
\mathfrak{Q} \underset{\beta}{E}\left[\left\|\beta^{*}\right\|-1 \beta^{*} \in \alpha\right]
$$

then

$$
\nu(\mathfrak{Q}) \leqq 8^{n} \lambda^{2 n}
$$

Proof. There being no difficulty in the event $\mathfrak{W}=0$, assume $\mathfrak{S} \neq 0$, recall the definition of $\mathfrak{Q}$, and from the finite nonvacuous family $\mathfrak{E}$ select such a member $b$ that

$$
8 \lambda \operatorname{diam} a<\left\|b^{*}\right\| \leqq\left\|\beta^{*}\right\| \quad \text { whenever } \beta \in \mathfrak{W} .
$$


Since the assumption diam $\alpha \leqq(8 \lambda)^{-1}$ clearly implies

$$
\|\| \beta^{*}\left\|^{-1} \beta^{*}-\right\| b^{*}\left\|^{-1} b^{*}\right\| \leqq(8 \lambda)^{-1} \quad \text { whenever } \beta \in \mathfrak{G}
$$

it is evident from Part IX that

$$
b^{\prime} \in \beta \quad \text { whenever } \beta \in \mathfrak{W} .
$$

Consequently Part VI assures us

$$
\lambda^{-1} \operatorname{diam} b \leqq \operatorname{diam} \beta \leqq 2 \operatorname{diam} b \quad \text { whenever } \beta \in \mathfrak{S} .
$$

In the light of Part III, (13), and (14) it may be seen that

$$
K_{\beta} \subset \beta \subset\left(\mathfrak{S}\left(b^{\prime}, 2 \operatorname{diam} b\right) \quad \text { whenever } \beta \in \mathfrak{W}\right. \text {. }
$$

With the aid of Part I, the definition of $K_{\beta}$ and (14) it may be inferred, for $\beta \in \mathfrak{W}$, that

$$
\mu\left(K_{\beta}\right)=\left\{(4 \lambda)^{-1} \operatorname{diam} \beta\right\}{ }^{n} v \geqq\left\{\left(4 \lambda^{2}\right)^{-1} \operatorname{diam} b\right\}^{n} v .
$$

From (16), Part VIII, (15), and Part I it now follows that

$$
\begin{aligned}
\left\{\left(4 \lambda^{2}\right)^{-1} \operatorname{diam} b\right\}^{n} v \nu(\mathfrak{W}) & =\sum_{\beta \in \mathfrak{\Phi}}\left\{\left(4 \lambda^{2}\right)^{-1} \operatorname{diam} b\right\}^{n} v \\
& \leqq \sum_{\beta \in \mathfrak{W}} \mu\left(K_{\beta}\right) \leqq \mu\left\{\left(\left(b^{\prime}, 2 \operatorname{diam} b\right)\right\}=\{2 \operatorname{diam} b\}^{n} v .\right.
\end{aligned}
$$

In accordance with this

$$
\nu(\mathfrak{W}) \leqq 8^{n} \lambda^{2 n} .
$$

PART XI. $\nu(\mathfrak{Q}) \leqq(512)^{n} \lambda^{3 n}$.

Proof. Let

$$
q=(32 \lambda)^{-1}
$$

and let $\mathfrak{F}$ be the family of all spheres of the form $\mathfrak{C}(z, q)$ where $z \in \mathfrak{C}(\theta, 1)$. Aided by 4.10 select such a disjointed subfamily $\mathfrak{B}$ of $\mathfrak{F}$ that each $\beta$ in $\mathfrak{F}$ intersects some $B$ in $\$$. Agree that

$$
\overline{\mathfrak{B}}=\underset{x}{E}[\|x-y\| \leqq q \text { for some } y \text { in } B]
$$

and check that

$$
\mathfrak{S}(\theta, 1) \subset \sum_{B \in G} \bar{B}
$$

and also that

$$
\operatorname{diam} \bar{B} \leqq 4 q=(8 \lambda)^{-1} \quad \text { whenever } B \in \mathcal{G}
$$

Clearly

$$
B \subset \mathfrak{S}(\theta, 2) \quad \text { whenever } B \in \mathbb{S} \text {; }
$$




$$
\begin{array}{rlr}
\mu(B) & =q^{n} v & \text { whenever } B \in \mathbb{S} ; \\
q^{n} v \nu(\mathbb{S}) & =\sum_{B \in \Theta} \mu(B) \leqq \mu\left\{\mathbb{S}(\theta, 2)=2^{n} v ;\right. & \\
\nu(\mathbb{S}) & \leqq 2^{n} q^{n}=2^{n}(32 \lambda)^{n}=64^{n} \lambda^{n} .
\end{array}
$$

Since, as may be gathered from the definition of $\mathfrak{Q}$ and from (17),

$$
\mathfrak{Q}=\mathfrak{Q} \underset{\beta}{E}\left[\left\|\beta^{*}\right\|^{-1} \beta^{*} \in \mathfrak{S}(\theta, 1)\right] \subset \sum_{B \in \mathfrak{S}} \mathfrak{Q} \underset{\beta}{E}\left[\left.\left\|\beta^{*}\right\|\right|^{-1} \beta^{*} \in \bar{B}\right]
$$

we conclude with the help of (18), Part X, and (19) that

$$
\begin{aligned}
\nu(\mathfrak{Q}) & \leqq \sum_{B \in \Theta} \nu\left(\mathfrak{Q} \underset{\beta}{E}\left[\left.\left\|\beta^{*}\right\|\right|^{-1} \beta^{*} \in \bar{B}\right)\right] \\
& \leqq \sum_{B \in \Theta} 8^{n} \lambda^{2 n}=\nu(\mathcal{S})(8)^{n} \lambda^{2 n} \\
& \leqq(64)^{n}(8)^{n} \lambda^{3 n}=(512)^{n} \lambda^{3 n} .
\end{aligned}
$$

Part XII. $\nu(\mathfrak{B}) \leqq(128)^{n} \lambda^{2 n}$.

Proof. If $a \neq \beta \in \mathfrak{B}$ and $y$ is any point in $K_{\beta}$ then in the light of the definition of $K_{\beta}$, Part VII, and the definition of $\mathfrak{P}$ it is seen that

$$
\begin{aligned}
\left\|y-a^{\prime}\right\| & \leqq\left\|y-\beta^{\prime}\right\|+\left\|\beta^{\prime}-a^{\prime}\right\| \leqq(4 \lambda)^{-1} \operatorname{diam} \beta+\left\|\beta^{\prime}-a^{\prime}\right\| \\
& \leqq(2 \lambda)^{-1} \operatorname{diam} \beta+\left\|\beta^{\prime}-a^{\prime}\right\| \leqq 2\left\|\beta^{\prime}-a^{\prime}\right\|=2\left\|\beta^{*}\right\| \leqq 16 \lambda \operatorname{diam} a .
\end{aligned}
$$

Thus, even though $\beta$ may equal $a$,

$$
K_{\beta} \subset \mathfrak{C}\left(a^{\prime}, 16 \lambda \operatorname{diam} a\right) \quad \text { whenever } \beta \in \mathfrak{B} .
$$

Since each member of $\mathfrak{B}$ is also a member of $\operatorname{circ} a$ we 5.8 know

$$
\operatorname{diam} a \leqq 2 \operatorname{diam} \beta \quad \text { for } \beta \in \Re .
$$

Accordingly for $\beta \in \mathfrak{B}$, we have

$$
\mu\left(K_{\beta}\right)=\left\{(4 \lambda)^{-1} \operatorname{diam} \beta\right\}^{n} v \geqq\left\{(8 \lambda)^{-1} \operatorname{diam} a\right\}^{n} .
$$

Bearing this and (20) in mind we infer

$$
\begin{aligned}
\left\{(8 \lambda)^{-1} \operatorname{diam} a\right\}^{n} v \nu(\mathfrak{B}) & =\sum_{\beta \in \mathfrak{B}}\left\{(8 \lambda)^{-1} \operatorname{diam} a\right\}^{n} v \\
& \leqq \sum_{\beta \in \mathfrak{B}} \mu\left(K_{\beta}\right) \leqq \mu\left\{\mathfrak{S}\left(a^{\prime}, 16 \lambda \operatorname{diam} a\right)\right\}=\{16 \lambda \operatorname{diam} a\}^{n} v
\end{aligned}
$$

Consequently

$$
\nu(\mathfrak{B}) \leqq(128)^{n} \lambda^{2 n} .
$$

PART XIII. $\nu(\Re \operatorname{circ} a) \leqq(9 \lambda)^{8 n}$.

Proof. Reference to the definitions of $\mathfrak{B}$ and $\mathfrak{Q}$ and to Parts XI and XII convinces us that 


$$
\begin{aligned}
\nu(\Re \operatorname{circ} a) & =\nu(\mathfrak{B}+\mathscr{Q})=\nu(\mathfrak{B})+\nu(\mathfrak{Q}) \\
& \leqq(512)^{n} \lambda^{3 n}+(128)^{n} \lambda^{2 n} \\
& \leqq(512)^{n} \lambda^{3 n}+(128)^{n} \lambda^{3 n} \\
& \leqq(512+128)^{n} \lambda^{3 n}=(640)^{n} \lambda^{3 n} \\
& <(729)^{n} \lambda^{3 n}=9^{3 n} \lambda^{3 n}=(9 \lambda)^{3 n} .
\end{aligned}
$$

5.10. ThEOREM. If $\lambda$ is a positive integer,

$$
\eta=(9 \lambda)^{3 n},
$$

$R$ is a diametrically restrained covering relation with range $\Re$,

and

$$
\sup _{\beta \in \Re} \operatorname{diam} \beta<\infty,
$$

$$
\operatorname{diam} \beta<\lambda \operatorname{hbrd}(z, \beta) \quad \text { whenever }(z, \beta) \in R,
$$

then there is such a sequence $\Gamma$ of countable disjointed families that

$$
\Re=\sum_{j=1}^{\eta} \Gamma_{j}
$$

Proof. To clear up any possible terminological misunderstanding I should like to point out in advance that the sequence $\Gamma$ whose existence is asserted is to have the property that for each non-negative integer $j$ no two different members of $\Gamma_{j}$ intersect. Taking advantage of 4.10 we define by induction a sequence $\Gamma$ for which

$$
\Gamma_{0}=0 ;
$$

if $j$ is any positive integer then $\Gamma_{j}$ is such a disjointed subfamily of

$$
\Re-\sum_{k=0}^{j-1} \Gamma_{k}
$$

that each member, $\beta$, of

$$
\Re-\sum_{k=0}^{j-1} \Gamma_{k}
$$

intersects some member $B$ of $\Gamma_{j}$ in such a way that $\operatorname{diam} \beta \leqq 2 \operatorname{diam} B$.

Evidently

$$
\Gamma_{i} \Gamma_{j}=0
$$

whenever $i$ and $j$ are different non-negative integers. Moreover for each nonnegative integer $j$, the family $\Gamma_{j}$ is countable because it is disjointed and consists of sets with inner points. 
In order to show that

$$
\Re=\sum_{j=1}^{\eta} \Gamma_{i}
$$

we assume that

$$
\Re \neq \sum_{i=1}^{n} \Gamma_{i}
$$

and proceed to a contradiction.

Because of (2) and the vacuity of $\Gamma_{0}$ the family

$$
\Re-\sum_{k=0}^{\eta} \Gamma_{k}
$$

is nonvacuous and from it we select a member $a$. If $j=1,2, \cdots, \eta$ then

$$
a \in \Re-\sum_{k=0}^{\eta} \Gamma_{k} \subset \Re-\sum_{k=0}^{1-1} \Gamma_{k}
$$

and our definition of $\Gamma$ tells us $a$ intersects some member $B$ of $\Gamma_{j}$ in such a way that $\operatorname{diam} a \leqq 2 \operatorname{diam} B$. It is accordingly 5.8 clear that

$$
\Gamma_{j} \operatorname{circ} a \neq 0
$$$$
\text { for } j=1,2, \cdots, \eta
$$

and hence 5.7 that

$$
\nu\left(\Gamma_{i} \operatorname{circ} a\right) \geqq 1 \quad \text { for } j=1,2, \cdots, \eta .
$$

In contradiction to 5.9 it now follows from (1), (3), and our hypotheses that

$$
\begin{aligned}
\nu(\Re \operatorname{circ} a) & \geqq \sum_{j=1}^{\eta} \nu\left(\Gamma_{j} \operatorname{circ} a\right) \\
& \geqq \sum_{j=1}^{\eta} 1=\eta=(9 \lambda)^{3 n} .
\end{aligned}
$$

5.11. ThEOREM. If $\phi \in \mathcal{U}$ and $F$ is a 5.6 star blanket then $F$ is $a \phi$ heavy 2.5 blanket.

Proof. Let $A$ be the domain of $F$, let $\mathfrak{F}$ be the spread of $F$, and let

$$
S_{\lambda}=\underset{x}{E}\left[\operatorname{limalsup}_{F(x) \exists \beta \rightarrow x} \frac{\operatorname{diam} \beta}{\operatorname{hbrd}(x, \beta)}<\lambda\right] \text {. }
$$

Noting that

$$
A=\sum_{\lambda=1}^{\infty} S_{\lambda}
$$


we divide the remainder of the proof into 4 parts.

PART I. If $\lambda$ is a positive integer, $B=S_{\lambda}$,

$$
\eta=(9 \lambda)^{n},
$$

and $\alpha$ is an open set then there is a countable disjointed subfamily $\Re$ of $\mathfrak{F}$ for which

$$
\sigma(\Omega) \subset \alpha, \quad \phi(B \alpha) \leqq \phi\{\sigma(\Omega\} .
$$

Proof. In keeping with (1) correlate with each $x$ in $B \alpha$ such a member $b_{x}$ of $F(x)$ that:

$$
b_{x} \subset \alpha
$$

and

$$
\operatorname{diam} b_{x}<\lambda \operatorname{hbrd}\left(x, b_{x}\right) .
$$

Let $Q$ consist of those pairs $\left(x, b_{x}\right)$ for which $x \in B \alpha$. Observe that $Q$ is a 4.4 covering relation with 4.3 domain $B \alpha$; let the 4.3 range of $Q$ be $\mathfrak{Q}$; check that

$$
\mathfrak{Q} \subset \mathfrak{F}
$$

and from (4) that

$$
\operatorname{diam} \beta<\lambda \text { hbrd }(z, \beta) \quad \text { whenever }(z, \beta) \in Q .
$$

Since $b_{x}$ is (3) contained in the bounded open set $\alpha$ we know

$$
\sigma(\mathfrak{Q}) \subset \alpha .
$$

and

$$
\sup _{\beta \in \mathfrak{O}} \operatorname{diam} \beta<\infty .
$$

Consequently 4.8 allows us to choose a diametrically restrained subrelation $R$ of $Q$ whose range covers $B \alpha$. Letting $\Re$ be the range of $R$ we are enabled by (6), (8) and 5.10 to select such a sequence $\Gamma$ of countable disjointed families that

$$
\Re=\sum_{j=1}^{\eta} \Gamma_{j} ;
$$

and, since $\Re$ covers $B \alpha$, we are enabled in turn by (9) to select an integer $k$ for which

$$
1 \leqq k \leqq \eta
$$

and

$$
\phi(B \alpha) \leqq \eta \phi\left\{\sigma\left(\Gamma_{k}\right)\right\} .
$$

Letting $\Omega=\Gamma_{k}$ we see at once that $\Re$ is a countable disjointed family for 
which

$$
\phi(B \alpha) \leqq \eta \phi\{\sigma(\Omega)\} .
$$

Moreover an appeal to (9), (5) and (7) helps us infer

$$
R \subset Q, \quad \Re \subset \Re \subset \mathfrak{Q} \subset \mathfrak{F}, \quad \sigma(\Re) \subset \sigma(\mathfrak{Q}) \subset \alpha .
$$

It is now evident that $\Omega$ has the properties demanded of it.

PART II. If $\lambda$ is a positive integer then $\mathfrak{F}$ has the $\phi$ Vitali property with respect to $S_{\lambda}$.

Proof. Let $B=S_{\lambda}, \eta=(9 \lambda)^{n}$, and from Part I and Theorem 3.4 learn that $\mathfrak{F}$ has the $\phi$ Vitali property with respect to $B$.

PART III. F has the $\phi$ Vitali property with respect to $A$.

Proof. Recall (2), and employ Part II, and Theorem 3.3.

PART IV. F is a $\phi$ heavy blanket.

Proof. Use Part III, take $\mathfrak{S}=0$ in 3.1 , and recall 2.5.

5.12. ThEOREM. If $\phi \in \mathcal{V}$ and $F$ is a star blanket then $F$ is a $\phi$ strong blanket.

Proof. Let $F^{\prime}$ be any 2.3 subblanket of $F$. Since $F^{\prime}$ is manifestly a star blanket we conclude from 5.11 that it is $\phi$ heavy.

Reference to 2.6 completes the proof.

5.13. TheOREM. If $F$ is a star blanket then $F$ is a perfect blanket.

Proof. Use 5.12 and 2.7.

5.14. Remark. According to 5.13 the differential properties enjoyed by perfect blankets in $\$ 2$ are shared by star blankets. We content ourselves here merely with the following consequence of 5.13, 2.24, and 2.17.

5.15. ThEOREM. Every star blanket is both perfect and universal.

The University of California, Berkeley, Calif. 